\title{
The Role of Human Immunodeficiency Virus (HIV) Asymptomatic Status When Starting Antiretroviral Therapy on Adherence and Treatment Outcomes and Implications for Test and Treat: The Swiss HIV Cohort Study
}

\begin{abstract}
Tracy R. Glass, ${ }^{1,2, \odot}$ Huldrych F. Günthard, ${ }^{3,4}$ Alexandra Calmy, ${ }^{5}$ Enos Bernasconi, ${ }^{6}$ Alexandra U. Scherrer, Manuel Battegay, ${ }^{2,8}$ Ana Steffen ${ }^{9}$ Jürg Böni, ${ }^{4}$ Sabine Yerly, ${ }^{10}$ Thomas Klimkait, ${ }^{11}$ Matthias Cavassini, ${ }^{12}$ and Hansjakob Furrer ${ }^{13}$; for the Swiss HIV Cohort Study

${ }^{1}$ Department of Medicine, Swiss Tropical and Public Health Institute, Basel, Switzerland, ${ }^{2}$ University of Basel, Basel, Switzerland, ${ }^{3}$ Division of Infectious Diseases and Hospital Epidemiology, University Hospital Zurich, Zurich, Switzerland, ${ }^{4}$ Institute of Medical Virology, University of Zurich, Zurich, Switzerland, ${ }^{5}$ Division of Infectious Diseases, University Hospital Geneva, Geneva, Switzerland, ${ }^{6}$ Division of Infectious Diseases, Regional Hospital Lugano, Lugano, Switzerland, ${ }^{7}$ Swiss HIV Cohort Data Center, Zurich, Switzerland, ${ }^{8}$ Division of Infectious Diseases, University Hospital Basel, Basel, Switzerland, ${ }^{9}$ Division of Infectious Diseases, Kantonsspital St Gallen, St Gallen, Switzerland, ${ }^{10}$ Laboratory of Virology, Division of Infectious Diseases, Geneva University Hospitals, Geneva, Switzerland, ${ }^{11}$ Department of Biomedicine, University of Basel, Basel, Switzerland, ${ }^{12}$ Division of Infectious Diseases, University Hospital Lausanne, Lausanne, Switzerland, and ${ }^{13}$ Department of Infectious Diseases, Bern University Hospital, University of Bern, Bern, Switzerland
\end{abstract}

Background. Since the advent of universal test-and-treat, more people living with human immunodeficiency virus (PLHIV) initiating antiretroviral therapy (ART) are asymptomatic with a preserved immune system. We explored the impact of asymptomatic status on adherence and clinical outcomes.

Methods. PLHIV registered in the Swiss HIV Cohort Study (SHCS) between 2003 and 2018 were included. We defined asymptomatic as Centers for Disease Control and Prevention stage A within 30 days of starting ART, non-adherence as any self-reported missed doses and viral failure as two consecutive viral load $>50$ copies/mL after $>24$ weeks on ART. Using logistic regression models, we measured variables associated with asymptomatic status and adherence and Cox proportional hazard models to assess association between symptom status and viral failure.

Results. Of 7131 PLHIV, 76\% started ART when asymptomatic and 1478 (22\%) experienced viral failure after a median of 1.9 years (interquartile range, 1.1-4.2). In multivariable models, asymptomatic PLHIV were more likely to be younger, men who have sex with men, better educated, have unprotected sex, have a HIV-positive partner, have a lower viral load, and have started ART more recently. Asymptomatic status was not associated with nonadherence (odds ratio, 1.03 [95\% confidence interval $\{\mathrm{CI}\}$, .93-1.15]). Asymptomatic PLHIV were at a decreased risk of viral failure (adjusted hazard ratio, 0.87 [95\% CI, .76-1.00]) and less likely to develop resistance $(14 \%$ vs $27 \%, P<.001)$ than symptomatic PLHIV.

Conclusions. Despite concerns regarding lack of readiness, our study found no evidence of adherence issues or worse clinical outcomes in asymptomatic PLHIV starting ART.

Keywords. HIV; antiretroviral therapy; asymptomatic; universal test and treat; clinical outcomes.

Many cohort studies have suggested a benefit of early treatment, and some guidelines already advocated in 2012 for starting treatment irrespective of CD4 cell count $[1,2]$. However, only after evidence from 2 randomized trials, Strategic Timing of Antiretroviral Therapy and Early Antiretroviral Treatment in HIV-infected Adults, did the World Health Organization recommend in September 2015 that people living with human

Received 25 November 2019; editorial decision 1 March 2020; accepted 9 March 2020; published online March 11, 2020.

Correspondence: T. R. Glass, Swiss Tropical and Public Health Institute, Socinstrasse 57, CH-4051 Basel, Switzerland (tracy.glass@swisstph.ch).

\section{Clinical Infectious Diseases ${ }^{\circledR} \quad$ 2020;XX(XX):1-9}

(C) The Author(s) 2020. Published by Oxford University Press for the Infectious Diseases Society of America. All rights reserved. For permissions, e-mail: journals.permissions@oup.com. DOI: 10.1093/cid/ciaa239 immunodeficiency virus (PLHIV) should begin antiretroviral therapy (ART) as soon as possible after diagnosis [3-6]. Prior to this, individual preference for timing of ART initiation varied widely, implying that shared decision making determined optimal start time [7].

Now, due to the Joint United Nations Programme on HIV/ AIDS goal of $90 \%$ of PLHIV knowing their human immunodeficiency virus (HIV) status [8], we have seen PLHIV not only starting ART earlier, but presenting earlier as well. Combined with the advent of a universal test-and-treat approach, a higher share of PLHIV initiating ART will be asymptomatic, leading to concerns regarding suboptimal adherence $[9,10]$. Although there remains limited experience with PLHIV starting ART at CD4 counts $>500$ cells $/ \mu \mathrm{L}$, starting ART with higher CD4 cell count has been shown to 
be a risk factor for treatment interruptions and viral failure [11-13]. Critics of the test-and-treat strategy suggest that it could lead to "over-testing, over-treatment, side-effects, resistance and potentially reduced autonomy of the individual in their choices of care" [14]. A mathematical modeling study in men who have sex with men (MSM) predicted that despite a $34 \%$ reduction in new infections, $19 \%$ reduction in deaths, and $39 \%$ reduction in new AIDS cases with test and treat, a near doubling of the prevalence of multidrug resistance $(9.1 \%$ compared to $4.8 \%$ ) by 2023 [15]. However, real-world data from the Swiss HIV Cohort Study (SHCS) do not support this bleak view, with evidence that emergence of drug resistance dramatically decreased over time [16] despite increasing numbers treated at higher CD4 levels $[2,17]$. A recent study from rural South Africa did not find an association between CD4 cell count at ART initiation and suboptimal adherence, and viral suppression at 12 months was 97\% [18]. A similar study looking at asymptomatic PLHIV in South Africa and Uganda found high adherence (measured with electronic monitoring) and viral suppression among those with earlystage infection [19].

There have been only a few key studies on PLHIV starting ART with high CD4 cell counts in sub-Saharan Africa $[18,20]$, and even fewer from resource-rich settings [13]. Given the concerns regarding a universal test-and-treat policy, we utilized the comprehensive data from the SHCS to address these important questions in a high-income setting. We assessed trends in starting ART over time and the potential impact of symptom status at ART initiation on adherence and treatment outcomes.

\section{METHODS}

The SHCS was established in 1988 and includes all consenting PLHIV 18 years or older [21]. Data on clinical and behavioral factors are collected every 6 months while laboratory data are collected every 3-6 months. The SHCS is representative of the Swiss epidemic, including $72 \%$ of those on ART and at least an estimated 50\% of all PLHIV [22, 23].

We considered PLHIV registered in SHCS between 2003 (when adherence was first measured) and 2018 for inclusion. We excluded PLHIV who started ART before 2000, those with HIV type 2 (HIV-2), and those who were pregnant at time of starting ART. Database closure was 30 April 2019.

\section{Adherence}

The SHCS adherence questionnaire was introduced in 2003 and contains 2 self-report questions addressing missed doses (daily, more than once a week, once a week, once every second week, once a month, never) and consecutive missed doses (yes, no) in the last 4 weeks. Classifying PLHIV as missing $0,1,2$, or $>2$ doses has shown to be predictive of virological failure and mortality [24].

\section{Treatment Outcomes}

Durability of first regimen was defined as time from starting ART until any change in ART regimen. Treatment interruption was defined as stopping ART for $>1$ week after ART initiation and categorized by length of treatment interruption in days (7-30, 31-60, 61-90, 91-180, >180). Planned treatment interruptions as part of clinical studies were excluded.

Virological suppression was defined as plasma HIV RNA $<50$ copies/mL after a maximum of 24 weeks on ART. Two thresholds were considered in the estimation of virological failure: plasma HIV type 1 (HIV-1) RNA $\geq 50$ and $\geq 200$ copies $/ \mathrm{mL}$. Virological failure defined as 2 consecutive viral loads above the threshold after achieving viral suppression or a minimum of 24 weeks on ART.

\section{Resistance}

The SHCS has a linked drug resistance database, which includes sequences from genotypic resistance tests performed by 4 authorized laboratories in Switzerland. More than 12000 sequences were generated retrospectively from the biobank to cover episodes of treatment failure and transmitted drug resistance, when drug resistance testing was not performed routinely [25]. Sequences are stored in a central database (SmartGene; Integrated Database Network System version 3.9.0). All laboratories performed population-based sequencing $[25,26]$.

Transmitted drug resistance defined as any surveillance drug resistance mutation detected in tests done within 30 days of starting ART or earlier [27]. Acquired drug resistance was defined as the occurrence of at least 1 new major mutation listed by the International Antiviral Society from samples $>30$ days after starting ART [28].

\section{Exposure}

The exposure was asymptomatic status of the PLHIV prior to starting ART. As the cohort does not record information on symptom status, but reported Centers for Disease Control and Prevention (CDC) stage since inception, we defined asymptomatic as CDC stage A until 30 days after starting ART.

\section{Confounders}

The following potential baseline (ART start) confounders are included in all models: age, gender, risk group for HIV infection (MSM vs heterosexual, injecting drug use, and other), higher education ( $>9$ years of schooling), white ethnicity, self-reported unprotected sex (previous 6 months), stable partnership (previous 6 months), living alone, psychiatric problems (treatment by psychiatrist in previous 6 months), legal problems (imprisonment within previous 6 months), viral load ( $-90 /+30$ days of ART initiation), and calendar year. In models for duration of first ART and viral failure, ART regimen and transmitted resistance were also included. In the model for viral failure, the average time between RNA measurements was included to 
address potential bias due to variation in timing of follow-up. Baseline CD4 cell count was not included as a confounder due to its high correlation with asymptomatic status.

\section{Statistical Analysis}

Baseline was date of ART start. Baseline characteristics were described according to symptom status using appropriate summary measures. We compared the treatment experience of PLHIV - such as duration of first ART and treatment interruptions-according to exposure group using $\chi^{2}$ tests or Wilcoxon rank-sum test of medians, as appropriate.

Multivariable logistic regression models were used to assess predictors of starting ART when asymptomatic. Generalized estimating equations estimated the association between symptom status at starting ART and self-reported nonadherence over time accounting for repeated questionnaires in the same individual. Multivariable Cox proportional hazards models estimated the association between starting ART when asymptomatic and (1) duration of first ART regimen and (2) viral failure. The proportional hazard assumption was assessed with Schoenfeld residuals. Prespecified potential confounders described above were included in all multivariable models. As a sensitivity analysis, we considered the subset of PLHIV starting ART from 2010 onward to account for the increase in later years of asymptomatic starting ART that also corresponded to better profile of ART drugs and shorter follow-up time. In a further sensitivity analysis, those with missing viral load outcome data were considered to have viral failure. Data are presented with odds ratios (ORs) or hazard ratios (HRs) and 95\% confidence intervals (CIs). Data were analysed using Stata version 14 software (StataCorp, College Station, Texas).

\section{RESULTS}

There were 8107 PLHIV newly registered in the SHCS between 2003 and 2018. Of these, 7131 were eligible for the study and 976 (12\%) were excluded; 318 did not start ART during the study period, 427 started ART prior to 2000, 219 were pregnant within 6 months of starting ART, and 12 had HIV-2 (Supplementary Figure 1).

\section{Trends in ART Start}

During the study period, PLHIV started ART a median of 9 (interquartile range [IQR], 3-94) weeks after being diagnosed with HIV. The time between HIV diagnoses and starting ART decreased from a median of 66 (IQR, 1-276) weeks for those diagnosed in 2000 to 2 (IQR, 1-4) weeks in 2018. There was a trend toward earlier start of ART over time; $33 \%$ started ART within 4 weeks of HIV diagnosis in 2000 compared to $75 \%$ in 2018 (Figure 1A). Median CD4 count at ART start was 279 (IQR, 156-420) cells/ $\mu \mathrm{L}$ and $16 \%$ started ART with CD4 count $>500$ cells $/ \mu \mathrm{L}$. There was a clear trend toward higher CD 4 cell counts at ART initiation over time (Figure 1B); however, 28\% started ART with a CD 4 count $<200$ cells/ $\mu \mathrm{L}$ in 2018.

\section{Symptom Status at ART Start}

Overall, 76\% started ART when asymptomatic and this increased over time from $62 \%$ in 2003 to $83 \%$ in $2017(P<.001)$. Median CD4 cell counts at ART initiation were significantly higher in asymptomatic PLHIV compared to symptomatic (median, 318 vs 123, respectively; $P<.001$ ). Integrase inhibitors were prescribed to $3 \%$ of PLHIV starting ART in 2009 compared to $80 \%$ in 2018 . Baseline characteristics according to symptom status are presented in Table 1.

Multivariable logistic regression models found that asymptomatic PLHIV starting ART were younger, were MSM, were more educated, had unprotected sex, had a stable HIV-positive partner, had a lower viral load, and started ART in a more recent calendar year (Supplementary Table 1).

\section{Adherence According to Symptom Status and CD4 Cell Count}

Self-reported adherence was recorded at each semiannual visit and PLHIV completed a median of 13 (IQR, 6-22) adherence reports. Adherence was high with PLHIV reporting never having missed a dose of ART on $87 \%$ of questionnaires. Trends in adherence over time remained relatively stable with slightly better adherence reported in the first year on ART and no difference between symptomatic and asymptomatic PLHIV (Supplementary Figure 2).

Correspondingly, in multivariable repeated-measures logistic regression models, asymptomatic status at ART start was not significantly associated with nonadherence during follow-up (OR, 1.03 [95\% CI, .93-1.15]; Table 2) but with other factors such as younger age, lower education levels, unprotected sex, lack of stable partnerships, and psychiatric problems (Supplementary Table 2). In sensitivity analyses, there remained no evidence of an association between asymptomatic status and nonadherence when considering the subset of PLHIV starting after 2009 (OR, 1.11 [95\% CI, .93-1.32]) or including only the first 2 years on ART (OR, 0.88 [95\% CI, .58-1.32]) (Table 2).

Similar to asymptomatic status, we did not find an association between CD4 count at ART initiation and nonadherence in univariable (per 100 cells/ $\mu \mathrm{L}$ increase: OR, 1.01 [95\% CI, .991.03]) or multivariable models (OR, 1.02 [95\% CI, .99-1.05]).

\section{Treatment Outcomes According to Symptom Status}

Treatment interruptions of 7 days or longer were not uncommon; $18 \%$ interrupted ART at any point during follow-up and $8 \%$ in the first year on ART (Table 3). Asymptomatic PLHIV had their first treatment interruption sooner after starting ART (median, 61 vs 85 weeks, $P=.002$ ), and the length of their first treatment interruption was longer than in symptomatic PLHIV (median, 26 vs 9 weeks, $P<.001$ ). However, these differences were no longer significant in the subset of PLHIV who started 

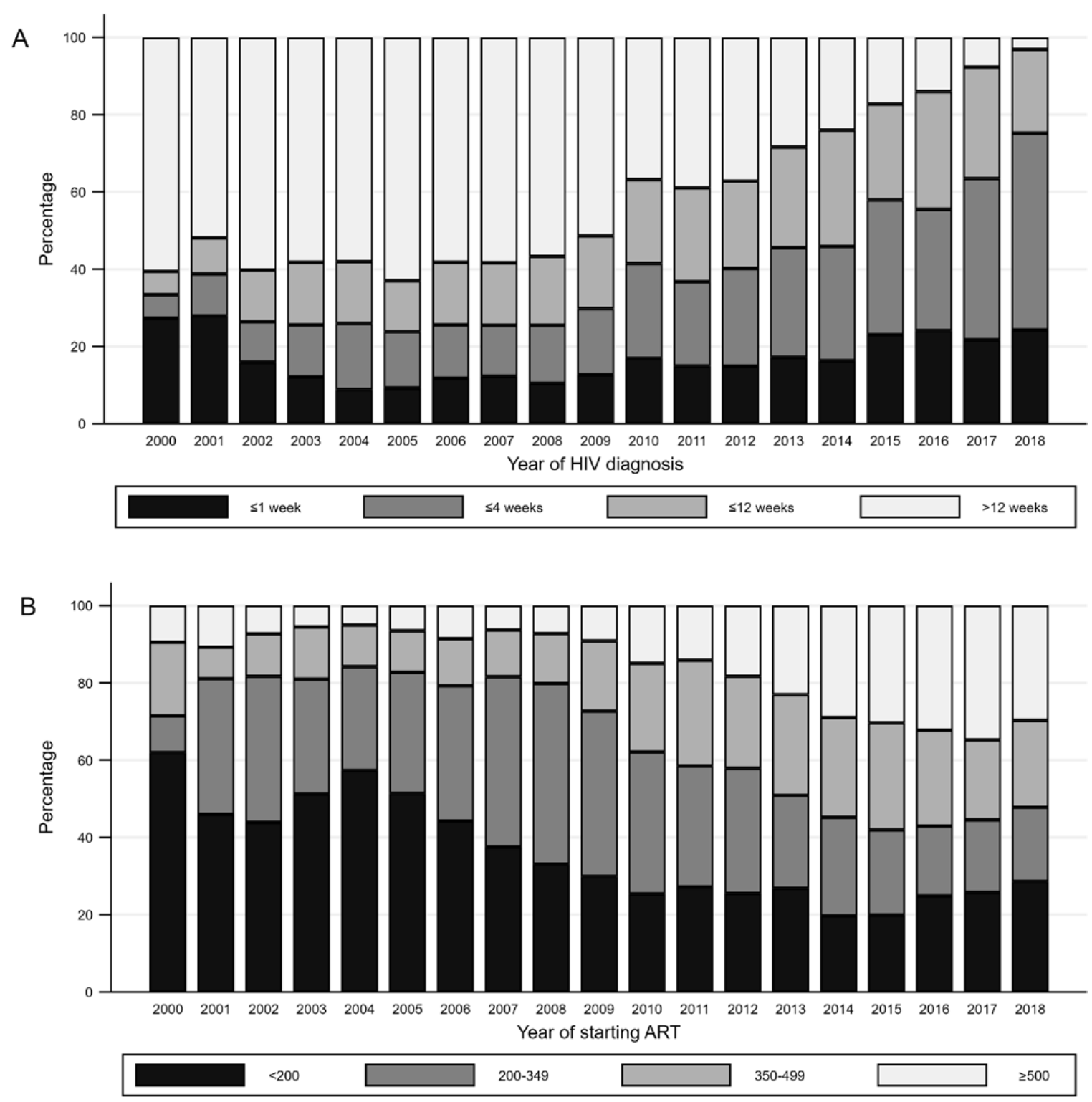

Figure 1. Trends in time until starting antiretroviral therapy (ART) in weeks by year of human immunodeficiency virus (HIV) diagnosis (A) and CD4 count (cells/ $\mathrm{LL}$ ) at time of starting ART by year of starting ART $(B)$.

ART after 2009 (median time to first interruption: 57 vs 73 weeks, $P=.28$; median length of interruption: 11 vs 10 weeks, $P=.30$ ). Sixty percent of PLHIV with a treatment interruption experienced confirmed viral failure, but this did not differ according to asymptomatic status.

Regimen changes were significantly less common in the asymptomatic PLHIV ( $86 \%$ vs $92 \%, P<.001$ ). Duration of first regimen was significantly longer in asymptomatic PLHIV (median, 79 vs 57 weeks, $P<.001$ ) (Figure $2 \mathrm{~A}$ ). These effects remained even in PLHIV starting ART after 2009.

Viral load outcomes were not assessable in 71 (1\%) PLHIV who stopped participation or died without having viral load recorded after starting ART. Of the 7060 evaluable PLHIV, median time to viral suppression was 18 weeks after starting ART (IQR, 10-32 weeks) (Table 3).

A further 313 (4\%) PLHIV did not have viral loads recorded after achieving viral suppression or 24 weeks on ART, leaving 6747 who could be evaluated for viral failure. Asymptomatic PLHIV were less likely to experience confirmed viral failure at a threshold of 50 copies $/ \mathrm{mL}(20 \%$ vs $27 \%, P<.001)$, and median time to virological failure was longer in the asymptomatic PLHIV (103 [IQR, 61-217] weeks) vs symptomatic PLHIV (93 [IQR, 50-218] weeks) ( $P=.02$; Figure 2B, Table 3 ).

In multivariable Cox proportional hazards models, asymptomatic PLHIV were at a decreased risk of confirmed viral failure at the threshold of RNA $<50$ copies/mL (HR, 0.87 [95\% CI, 


\begin{tabular}{|c|c|c|c|}
\hline & Symptomatic & Asymptomatic & Total \\
\hline Characteristic & $(n=1862)$ & $(n=5269)$ & $(N=7131)$ \\
\hline Age, y, median (IQR) & $41(33-50)$ & $37(30-45)$ & $38(31-46)$ \\
\hline Male sex & $1322(71)$ & $4156(79)$ & $5478(77)$ \\
\hline White & $1324(71)$ & $3841(73)$ & $5165(73)$ \\
\hline Education $<9$ y & $497(27)$ & $1121(21)$ & $1618(23)$ \\
\hline \multicolumn{4}{|l|}{ Risk group for HIV infection } \\
\hline MSM & $643(37)$ & $2974(59)$ & $3617(53)$ \\
\hline Heterosexual & $933(53)$ & $1739(34)$ & 2672 (39) \\
\hline Injection drug use & $133(8)$ & $296(6)$ & $429(6)$ \\
\hline Other & $51(3)$ & $65(1)$ & $116(2)$ \\
\hline \multicolumn{4}{|l|}{ Stable partnership ${ }^{a}$} \\
\hline No stable partner & $776(44)$ & $2081(42)$ & $2857(42)$ \\
\hline Stable partner, unknown HIV status & $300(17)$ & $651(13)$ & $951(14)$ \\
\hline Stable partner, HIV negative & $470(27)$ & $1365(27)$ & $1835(27)$ \\
\hline Stable partner, HIV positive & $220(12)$ & $876(18)$ & $1096(16)$ \\
\hline \multicolumn{4}{|l|}{ Sexual behavior ${ }^{a}$} \\
\hline No partner/protected sex & $1508(85)$ & $3745(75)$ & $5253(77)$ \\
\hline Reported unprotected sex & $238(13)$ & $1133(23)$ & $1371(20)$ \\
\hline Refused to answer & $6(0)$ & $31(1)$ & $37(1)$ \\
\hline Unknown/missing & $27(2)$ & $102(2)$ & $129(2)$ \\
\hline Living alone $^{a}$ & $680(38)$ & 1965 (39) & 2645 (39) \\
\hline Receiving psychiatric treatment ${ }^{\mathrm{a}}$ & $214(12)$ & $704(14)$ & $918(14)$ \\
\hline Legal problems (including imprisonment) ${ }^{a}$ & $20(1)$ & $89(2)$ & $109(2)$ \\
\hline \multicolumn{4}{|l|}{ Initial ART regimen } \\
\hline NNRTI & $707(38)$ & 1979 (38) & $2686(38)$ \\
\hline Pl-based & $904(49)$ & $2093(40)$ & $2997(42)$ \\
\hline Integrase inhibitor & $202(11)$ & $1037(20)$ & 1239 (17) \\
\hline Triple nucleoside/Fl/other & $49(3)$ & $160(3)$ & 209 (3) \\
\hline $\mathrm{CD} 4$ count, cells/ $\mathrm{\mu L}$, median (IQR) & $123(36-269)$ & $318(217-459)$ & $279(156-420)$ \\
\hline \multicolumn{4}{|l|}{ CD4 count category, cells $/ \mu \mathrm{L}$} \\
\hline$<200$ & $1060(64)$ & $920(21)$ & 1980 (33) \\
\hline $200-349$ & $338(20)$ & $1577(36)$ & 1915 (32) \\
\hline $350-499$ & $158(10)$ & $999(23)$ & $1157(19)$ \\
\hline$\geq 500$ & $104(6)$ & $877(20)$ & $981(16)$ \\
\hline HIV RNA, log copies/mL, median (IQR) & $11(10-13)$ & $11(9-12)$ & $11(9-12)$ \\
\hline \multicolumn{4}{|l|}{ HIV RNA category, copies/mL } \\
\hline$<10000$ & $337(20)$ & $1310(30)$ & $1647(28)$ \\
\hline $10000-49,999$ & $281(17)$ & $1127(26)$ & $1408(24)$ \\
\hline$\geq 50000$ & $1046(63)$ & $1864(43)$ & $2910(49)$ \\
\hline
\end{tabular}

Data are presented as no. (\%) unless otherwise indicated.

Abbreviations: ART, antiretroviral therapy; FI, fusion inhibitor; HIV, human immunodeficiency virus; IQR, interquartile range; MSM, men who have sex with men; NNRTI, nonnucleoside reverse transcriptase inhibitor; $\mathrm{Pl}$, protease inhibitor.

an the previous 6 months.

.76-1.00], $P=.05$; Supplementary Table 3 ). In sensitivity analyses considering the subset of PLHIV starting ART from 2010 onward, the association was even stronger (HR, $0.63[95 \% \mathrm{CI}$, $.49-.82], P<.001$; Table 2).

For the threshold of RNA $<200$ copies/mL, the association between asymptomatic status and viral failure was not significant in multivariable models (HR, 1.14 [95\% CI, .95-1.37], $P=.151$; Supplementary Table 3). In the subset of PLHIV starting ART from 2010 onward, the association was significant in univariable but not multivariable models (HR, 0.81 [95\% CI, .53-1.23], $P=.317$; Table 2).
More than $71 \%(n=5073)$ of PLHIV had at least 1 resistance test done prior to or within 30 days of starting ART. Transmitted resistance was found in 13\% $(\mathrm{n}=656)$ : $5 \%$ to nucleoside reverse transcriptase inhibitors (NRTIs; 240/5049), 7\% to nonnucleoside reverse transcriptase inhibitors (NNRTIs; $359 / 5049$ ), $3 \%$ to protease inhibitors (PI; 141/5055) and $<0.1 \%$ to integrase inhibitors (1/1543). After starting ART, 1025 were tested for resistance $>30$ days after starting ART. Of these, 641 (63\%) had a baseline resistance test and could be evaluated for newly acquired resistances of which 185 (29\%) were detected: $22 \%$ to NRTIs (142/636), $18 \%$ to NNRTIs (114/636), $3 \%$ to PIs 
Table 2. Regression Models for the Association Between Asymptomatic Status and Self-reported Nonadherence to Antiretroviral Therapy and Clinical Outcomes

\begin{tabular}{|c|c|c|c|}
\hline Characteristic & Univariable & Multivariable & $P$ Value \\
\hline \multicolumn{4}{|l|}{ Outcome: Self-reported nonadherence ${ }^{a}$} \\
\hline 2003-2018 & $1.03(.95-1.13)$ & $1.03(.93-1.15)$ & .576 \\
\hline Subset starting ART 2010-2018 & $1.17(1.02-1.36)$ & $1.11(.93-1.32)$ & .246 \\
\hline First 2 years on ART & $1.07(.77-1.48)$ & $0.88(.58-1.32)$ & .535 \\
\hline \multicolumn{4}{|l|}{ Outcome: Confirmed viral failure ${ }^{b}$} \\
\hline \multicolumn{4}{|c|}{ Two consecutive HIV RNA > 50 copies $/ \mathrm{mL}$} \\
\hline $2003-2018$ & $0.73(.66-.82)$ & $0.87(.76-1.00)$ & .05 \\
\hline With missing outcome excluded & $0.74(.66-.82)$ & $0.87(.76-1.00)$ & .05 \\
\hline With missing outcome $=$ failure & $0.78(.71-.86)$ & $0.87(.76-1.00)$ & .05 \\
\hline Subset starting ART 2010-2018 & $0.52(.42-.64)$ & $0.63(.49-.82)$ & $<.001$ \\
\hline \multicolumn{4}{|c|}{ Two consecutive HIV RNA > 200 copies $/ \mathrm{mL}$} \\
\hline 2003-2018 & $0.93(.81-1.08)$ & $1.14(.95-1.37)$ & .149 \\
\hline With missing outcome excluded & $0.94(.81-1.08)$ & $1.14(.95-1.37)$ & .149 \\
\hline With missing outcome $=$ failure & $0.96(.85-1.09)$ & $1.14(.95-1.37)$ & .149 \\
\hline Subset starting ART 2010-2018 & $0.61(.43-.86)$ & $0.81(.53-1.23)$ & .316 \\
\hline
\end{tabular}

Abbreviations: ART, antiretroviral therapy; HIV, human immunodeficiency virus.

${ }^{a}$ Generalized estimating equation models to account for the clustering from repeated measures of self-reported nonadherence in the same individual. Odds ratios and $95 \%$ confidence intervals (Cls) for the association between starting ART when asymptomatic and any self-reported missed doses of ART. The full model is reported in Supplementary Table 2 .

${ }^{b}$ Cox proportional hazards model for time to virological failure. Hazard ratios and $95 \%$ Cls for the association between starting ART when asymptomatic and confirmed virological failure. The full model is reported in Supplementary Table 3.

(20/632), and $6 \%$ to integrase inhibitors (4/63). Asymptomatic PLHIV were less likely to develop resistance (24\% vs $37 \%$, $P<.001)$ even in the subset who started ART after $2009(12 \%$ vs $24 \%, P=.02$ ).

\section{DISCUSSION}

Our study found no evidence to suggest that asymptomatic PLHIV struggle with adherence to ART or experience worse treatment outcomes compared to symptomatic PLHIV starting ART. On the contrary, asymptomatic PLHIV reported a similar number of missed doses of ART and treatment interruptions, along with a longer duration of their first ART regimen, and lower rates of viral failure and acquired resistance. However, these univariable associations potentially could have been confounded by the increase of asymptomatic PLHIV in recent years, which corresponded to both shorter time on ART and simpler, more tolerable regimens. After adjusting for a comprehensive list of confounders, asymptomatic PLHIV did not report more nonadherence and remained less likely to experience viral failure at the threshold of RNA $<50$ copies $/ \mathrm{mL}$. These results are particularly encouraging considering the already low rates of virologic failure and emergence of drug resistance in the cohort [29].

After 2008, we found clear trends in PLHIV starting ART more quickly and with higher CD4 cell counts. This increase coincided with the release of the Swiss statement postulating the near zero risk of PLHIV with suppressed viral load passing the virus on to uninfected partners [30] and with new ART treatment guidelines suggesting starting ART when CD4 counts fell below 500 cells/ $\mu \mathrm{L}$ [31]. Correspondingly, asymptomatic PLHIV starting ART were younger, were MSM, had unprotected sex, and were in a stable partnership. Starting ART within 4 weeks of diagnosis increased dramatically over time to $75 \%$ in 2018 while those starting within 1 week remained relatively stable at $24 \%$. As in many other countries, late presentation to care and late ART initiation remain a concern in Switzerland with little progress made since 2008 when almost one-third of PLHIV started ART with a CD4 count $<200$ cells/ $\mu \mathrm{L}[23,32-34]$.

In the subset of PLHIV who interrupted ART after initiation, asymptomatic PLHIV stopped earlier and for a longer time than symptomatic PLHIV. As this effect dissipated in those starting ART in later calendar years, this observed difference was likely reflective of an earlier era with less tolerable ART regimens and a corresponding lack of willingness on the part of asymptomatic PLHIV to endure side effects of treatment [35]. Results from the Treatment as Prevention (TasP) trial in South Africa, where PLHIV criteria for initiating ART were randomly assigned, starting ART at higher CD4 cell counts was not associated with suboptimal adherence [18]. Despite significant structural barriers, Haberer and colleagues found high median adherence and viral suppression at 12 months (89\% and 90\%, respectively, in Uganda; $76 \%$ and 86\%, respectively, in South Africa) among asymptomatic men and nonpregnant women initiating ART [19]. Among other factors, poor adherence was associated with trouble coping with HIV diagnosis. Newly diagnosed and asymptomatic PLHIV may not feel empowered to delay ART start, with clear evidence favoring immediate start, and it will be important to identify and support this potentially vulnerable 


\begin{tabular}{|c|c|c|c|}
\hline & Symptomatic & Asymptomatic & Total \\
\hline Characteristic & $(n=1862)$ & $(n=5269)$ & $(N=7131)$ \\
\hline Years on ART, median (IQR) & $8.1(3.8-11.6)$ & $6.4(3.2-9.8)$ & $6.8(3.3-10.3)$ \\
\hline \multicolumn{4}{|l|}{ Adherence } \\
\hline Adherence questionnaires, median (IQR) & $17(8-26)$ & $12(6-21)$ & $13(6-22)$ \\
\hline \multicolumn{4}{|l|}{ Worst reported missed doses in year 1} \\
\hline 0 missed doses & $1205(82)$ & $3196(82)$ & $4401(82)$ \\
\hline 1 missed dose & $161(11)$ & $456(12)$ & $617(12)$ \\
\hline 2 missed doses & $49(3)$ & $112(3)$ & $161(3)$ \\
\hline$\geq 3$ missed doses & $52(4)$ & $116(3)$ & $168(3)$ \\
\hline \multicolumn{4}{|l|}{ Treatment interruptions } \\
\hline Any treatment interruption & $335(18)$ & $963(18)$ & $1298(18)$ \\
\hline During year 1 & $128(7)$ & $428(8)$ & $556(8)$ \\
\hline Time until first treatment interruption, wk, median (IQR) & $85(19-233)$ & $61(14-167)$ & $66(16-188)$ \\
\hline Length of first treatment interruption, wk, median (IQR) & $9(3-35)$ & $26(6-104)$ & $20(5-87)$ \\
\hline \multicolumn{4}{|l|}{ Category of length of interruption, $d$} \\
\hline$\leq 30$ & $96(29)$ & $166(17)$ & $262(20)$ \\
\hline $31-60$ & $67(20)$ & $109(11)$ & $176(14)$ \\
\hline $61-90$ & $28(8)$ & $77(8)$ & $105(8)$ \\
\hline $90-180$ & $42(13)$ & $109(11)$ & $151(12)$ \\
\hline$>180$ & $102(30)$ & $502(52)$ & $604(47)$ \\
\hline Duration of first ART regimen, wk, median (IQR) & $57(12-162)$ & $79(22-187)$ & $74(20-181)$ \\
\hline \multicolumn{4}{|l|}{ Viral load } \\
\hline Viral suppression ${ }^{a}(n=7060)$ & $1794(97)$ & $5103(98)$ & $6897(98)$ \\
\hline Within 24 wk $(n=4048)$ & $1065(64)$ & $3272(74)$ & $4337(71)$ \\
\hline Time to viral suppression, wk, median (IQR) & $20(12-33)$ & $17(9-32)$ & $18(10-32)$ \\
\hline \multicolumn{4}{|l|}{ HIV-1 RNA $\geq 50$ copies/mL $(n=6747)$} \\
\hline Viral failure $^{\mathrm{b}}$ & $484(27)$ & $994(20)$ & $1478(22)$ \\
\hline Time to viral failure, wk, median (IQR) & $93(50-218)$ & $103(61-217)$ & $99(57-217)$ \\
\hline \multicolumn{4}{|l|}{ HIV-1 RNA $\geq 200$ copies/mL ( $n=6747)$} \\
\hline Viral failure $^{b}$ & $266(15)$ & $651(13)$ & $917(14)$ \\
\hline Time to viral failure, wk, median (IQR) & $149(80-280)$ & $115(70-248)$ & $122(71-260)$ \\
\hline \multicolumn{4}{|l|}{ Resistance } \\
\hline Transmitted resistance $(n=5073)$ & $180(14)$ & $4603(12)$ & $640(13)$ \\
\hline NRTI & $70(5)$ & $170(5)$ & $240(5)$ \\
\hline NNRTI & $108(8)$ & $251(7)$ & $359(7)$ \\
\hline $\mathrm{Pl}$ & $43(3)$ & $98(3)$ & $141(3)$ \\
\hline Integrase inhibitor & $0(0)$ & $1(0)$ & $1(0)$ \\
\hline Newly acquired resistance $(n=641)$ & $90(37)$ & $95(24)$ & $185(29)$ \\
\hline NRTI & $75(31)$ & $67(17)$ & $142(22)$ \\
\hline NNRTI & $55(23)$ & $59(15)$ & 114 (18) \\
\hline $\mathrm{Pl}$ & $10(4)$ & $10(3)$ & $20(3)$ \\
\hline Integrase inhibitor & $2(7)$ & $2(6)$ & $4(6)$ \\
\hline
\end{tabular}

Data are presented as no. (\%) unless otherwise indicated.

Abbreviations: ART, antiretroviral therapy; HIV-1, human immunodeficiency virus type 1; IQR, interquartile range; NNRTI, nonnucleoside reverse transcriptase inhibitor; NRTI, nucleoside reverse transcriptase inhibitor; $\mathrm{Pl}$, protease inhibitor.

airal suppression: viral load $<50$ copies $/ \mathrm{mL}$.

${ }^{b}$ Viral failure: 2 consecutive HIV-RNA viral loads above the stated threshold after achieving viral suppression.

subset. Wagner and colleagues are conducting a trial to assess readiness to start ART on adherence that may shed light on this important issue [36].

Our study has several strengths and limitations. The SHCS is known for comprehensive data collection in a real-world setting over many years, allowing us to assess patterns in starting ART and treatment outcomes before and after start of test and treat. We assessed many outcomes, including repeated adherence and resistance, often not measured in cohorts. On the other hand, the SHCS does not record key variables of interest, such as reported symptoms or reason for starting ART. In addition, adherence data are self-reported and susceptible to underreporting due to social desirability bias compared to more objective measures of adherence [37, 38]. However, this 

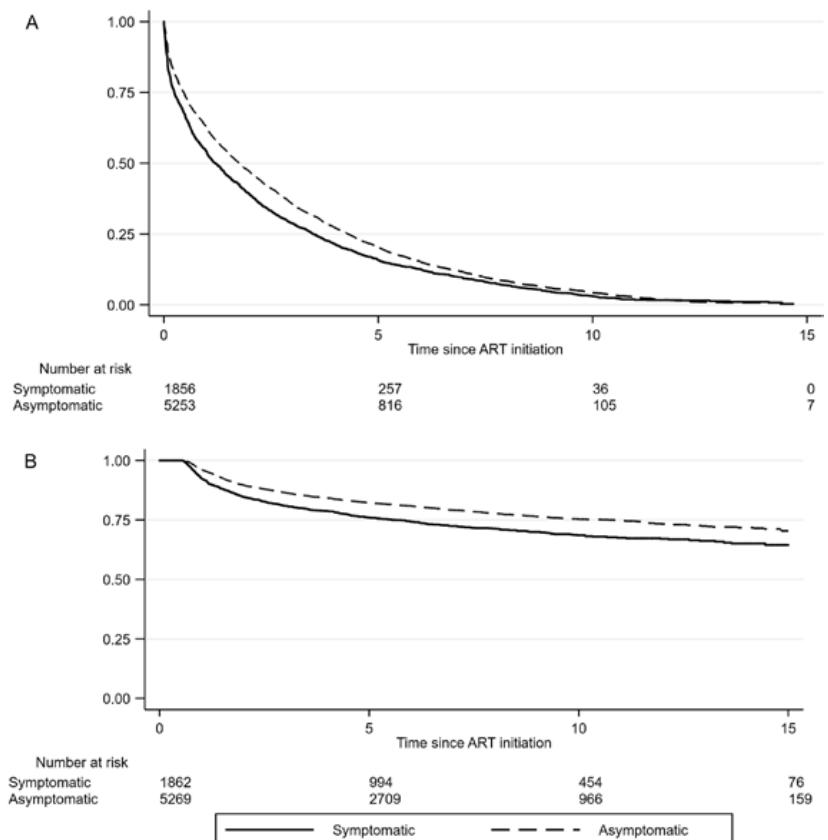

Figure 2. Kaplan-Meier curves for time to first change of antiretroviral therapy (ART) regimen $(A)$ and time to confirmed viral failure (2 consecutive human immunodeficiency virus RNA levels $>50$ copies $/ \mathrm{mL}$ ) by asymptomatic status $(B)$.

adherence measure correlates well with treatment outcomes $[24,39]$. The "asymptomatic" classification is based solely on the CDC stage and may potentially misclassify true symptom status in some cases. Overall, our conclusions were robust to the many sensitivity analyses conducted.

This is the first study to report comprehensive clinical picture of asymptomatic PLHIV starting ART in a high-income setting. The data are encouraging, and there is no reason to suspect changes given the potent and tolerable drugs currently available. However we should not become complacent and should continue to study readiness, adherence, and symptom management. Especially important is reducing late presentation through earlier diagnosis and engagement into care. With additional cost and resources required to treat PLHIV earlier, differentiated care models are especially important for monitoring newly diagnosed PLHIV starting ART and providing targeted support for vulnerable groups-younger, less educated, and lacking social support.

\section{Supplementary Data}

Supplementary materials are available at Clinical Infectious Diseases online. Consisting of data provided by the authors to benefit the reader, the posted materials are not copyedited and are the sole responsibility of the authors, so questions or comments should be addressed to the corresponding author.

\section{Notes}

Acknowledgments. The authors thank the participants of the Swiss HIV Cohort Study (SHCS); the physicians and study nurses for excellent patient care; the resistance laboratories for high-quality genotyping drug resistance testing; SmartGene (Zug, Switzerland) for technical support;
Alexandra Scherrer, Susanne Wild, and Anna Traytel, from the SHCS data center, for data management; and Danièle Perraudin and Marianne Amstad for administration. The current members of the SHCS are Aebi-Popp K., Anagnostopoulos A., Battegay M., Bernasconi E., Böni J., Braun D. L., Bucher H. C., Calmy A., Cavassini M., Ciuffi A., Dollenmaier G., Egger M., Elzi L., Fehr J., Fellay J., Furrer H., Fux C. A., Günthard H. F. (President of the SHCS), Haerry D. (deputy of "Positive Council"), Hasse B., Hirsch H. H., Hoffmann M., Hösli I., Huber M., Kahlert C. R. (Chairman of the Mother and Child Substudy), Kaiser L., Keiser O., Klimkait T., Kouyos R. D., Kovari H., Ledergerber B., Martinetti G., Martinez de Tejada B., Marzolini C., Metzner K. J., Müller N., Nicca D., Paioni P., Pantaleo G., Perreau M., Rauch A. (Chairman of the Scientific Board), Rudin C., Scherrer A. U. (Head of Data Centre), Schmid P., Speck R., Stöckle M. (Chairman of the Clinical and Laboratory Committee), Tarr P., Trkola A., Vernazza P., Wandeler G., Weber R., Yerly S. The SHCS was approved by the ethics committees of the participating institutions (Kantonale Ethikkommission Bern, Ethikkommission des Kantons St Gallen, Comité Départemental d’Éthique des Spécialités Médicales et de Médicine Communataire et de Premier Recours, Kantonale Ethikkommission Zürich, Repubblica et Cantone Ticino-Comitato Ethico Cantonale, Commission Cantonale d'Éthique de la Recherche sur l'Être Humain, Ethikkommission beider Basel for the SHCS and Kantonale Ethikkommission Zürich for the ZPHI), and written informed consent was obtained from all participants.

Financial support. This work was supported within the framework of the Swiss HIV Cohort Study (SHCS) (project 799), supported by the Swiss National Science Foundation (grant numbers 177499 and 179 571); by the Swiss HIV Cohort Research Foundation; and by the Yvonne-Jacob Foundation.

Potential conflicts of interest. T. R. G. has received academic grants from Gilead Sciences; and fees for data and safety monitoring board membership from Janssen, Novartis, and Baxalta. H. F. G. has received unrestricted research grants from Gilead Sciences, fees for data and safety monitoring board membership from Merck; consulting/advisory board membership fees from Gilead Sciences, ViiV, Sandoz, and Mepha; and grants from the National Institutes of Health. E. B. reports support to his institution for advisory boards and/or travel grants from Gilead, MSD, ViiV, AbbVie, Sandoz, and Pfizer. H. F's institution has received unrestricted educational grants from Gilead Sciences, ViiV, AbbVie, MSD, Janssen, and Sandoz paid to the institution. A. C. reports financial support for the day hospital to Geneva University Hospital (HIV/ AIDS Unit) from MSD, AbbVie, Gilead, and ViiV and unrestricted educational grants from MSD, ViiV, and Gilead. M. C. reports research grants from Gilead and ViiV and payments for expert opinion from AbbVie, Gilead, MSD, ViiV, and Sandoz. All other authors report no potential conflicts of interest. All authors have submitted the ICMJE Form for Disclosure of Potential Conflicts of Interest. Conflicts that the editors consider relevant to the content of the manuscript have been disclosed.

\section{References}

1. Thompson MA, Aberg JA, Hoy JF, et al. Antiretroviral treatment of adult HIV infection. JAMA 2012; 308:387-402.

2. Günthard HF, Aberg JA, Eron JJ, et al. Antiretroviral treatment of adult HIV infection: 2014 recommendations of the International Antiviral Society-USA Panel. JAMA 2014; 312:410-25.

3. World Heath Organization. Guideline on when to start antiretroviral therapy and on pre-exposure prophylaxis for HIV. 2015. Available at: http://www.who.int/hiv/ pub/guidelines/earlyrelease-arv/en/. Accessed 15 October 2015.

4. Cohen MS, Chen YQ, McCauley M, et al. Prevention of HIV-1 infection with early antiretroviral therapy. N Engl J Med 2011; 365:493-505.

5. Article O. A trial of early antiretrovirals and isoniazid preventive therapy in Africa. N Engl J Med 2015; 373:808-22.

6. Diseases I. Initiation of antiretroviral therapy in early asymptomatic HIV infection. N Engl J Med 2015; 373:795-807.

7. Locadia M, van Grieken RA, Prins JM, de Vries HJ, Sprangers MA, Nieuwkerk PT Patients' preferences regarding the timing of highly active antiretroviral therapy initiation for chronic asymptomatic HIV-1 infection. Antivir Ther 2006; 11:335-41.

8. Joint United Nations Programme on HIV/AIDS. 90-90-90 An ambitious treatment target to help end the AIDS epidemic. 2014:40. Available at: http://www unaids.org/sites/default/files/media_asset/90-90-90_en.pdf. Accessed 30 October 2014. 
9. McNairy ML, El-Sadr WM. Antiretroviral therapy for the prevention of HIV transmission: what will it take? Clin Infect Dis 2014; 58:1003-11.

10. Lockman S, Sax P. Treatment-for-prevention: clinical considerations. Curr Opin HIV AIDS 2012; 7:131-9.

11. Mills EJ, Funk A, Kanters S, et al. Long-term health care interruptions among HIV-positive patients in Uganda. J Acquir Immune Defic Syndr 2013; 63:23-7.

12. Adakun SA, Siedner MJ, Muzoora C, et al. Higher baseline CD4 cell count predicts treatment interruptions and persistent viremia in patients initiating ARVs in rural Uganda. J Acquir Immune Defic Syndr 2013; 62:317-21.

13. Torian L V, Xia Q. Achievement and maintenance of viral suppression in persons newly diagnosed with HIV, New York City, 2006-2009: using population surveillance data to measure the treatment part of "test and treat." J Acquir Immune Defic Syndr 2013; 63:379-86.

14. Garnett GP, Baggaley RF. Treating our way out of the HIV pandemic: could we, would we, should we? Lancet 2009; 373:9-11.

15. Sood N, Wagner Z, Jaycocks A, Drabo E, Vardavas R. Test-and-treat in Los Angeles: a mathematical model of the effects of test-and-treat for the population of men who have sex with men in Los Angeles County. Clin Infect Dis 2013; 56:1789-96.

16. Scherrer AU, von Wyl V, Yang WL, et al. Emergence of acquired HIV-1 drug resistance almost stopped in Switzerland: a 15-year prospective cohort analysis. Clin Infect Dis 2016; 62:1310-7.

17. Günthard HF, Saag MS, Benson CA, et al. Antiretroviral drugs for treatment and prevention of HIV infection in adults. JAMA 2016; 316:191.

18. Iwuji C, McGrath N, Calmy A, et al. Universal test and treat is not associated with sub-optimal antiretroviral therapy adherence in rural South Africa: the ANRS 12249 TasP trial. J Int AIDS Soc 2018; 21:1-11.

19. Haberer JE, Bwana BM, Orrell C, et al. ART adherence and viral suppression are high among most non-pregnant individuals with early-stage, asymptomatic HIV infection: an observational study from Uganda and South Africa. J Int AIDS Soc 2019; 22:e25232.

20. Eshleman SH, Wilson EA, Zhang XC, et al. Virologic outcomes in early antiretroviral treatment: HPTN 052. HIV Clin Trials 2017; 18:100-9.

21. Schoeni-Affolter F, Ledergerber B, Rickenbach M, et al. Cohort profile: the Swiss HIV Cohort Study. Int J Epidemiol 2010; 39:1179-89.

22. Kohler P, Schmidt AJ, Cavassini M, et al. The HIV care cascade in Switzerland: reaching the UNAIDS/WHO targets for patients diagnosed with HIV. AIDS 2015; 29:2509-15.

23. Hachfeld A, Ledergerber B, Darling K, et al. Reasons for late presentation to HIV care in Switzerland. J Int AIDS Soc 2015; 18:1-8.

24. Glass TR, Sterne JA, Schneider MP, et al. Self-reported nonadherence to antiretroviral therapy as a predictor of viral failure and mortality. AIDS 2015;29:2195-200.
25. Yang W-L, Kouyos R, Scherrer AU, et al. Assessing the paradox between transmitted and acquired HIV type 1 drug resistance mutations in the Swiss HIV Cohort Study from 1998 to 2012. J Infect Dis 2015; 212:28-38.

26. von Wyl V. Emergence of HIV-1 drug resistance in previously untreated patients initiating combination antiretroviral treatment: a comparison of different regimen types. Arch Intern Med 2007; 167:1782.

27. Bennett DE, Camacho RJ, Otelea D, et al. Drug resistance mutations for surveillance of transmitted HIV-1 drug-resistance: 2009 update. PLoS One 2009; $4: e 4724$.

28. Wensing AM, Calvez V, Günthard HF, et al. 2017 update of the drug resistance mutations in HIV-1. Top Antivir Med 2016; 24:132-3.

29. Abela IA, Scherrer AU, Böni J, et al. Emergence of drug resistance in the Swiss HIV Cohort Study under potent antiretroviral therapy is observed in socially disadvantaged patients. Clin Infect Dis 2020; 70:297-303.

30. Vernazza P, Bernard EJ. HIV is not transmitted under fully suppressive therapy: the Swiss Statement-eight years later. Swiss Med Wkly 2016; 146:w14246.

31. Thompson MA, Aberg JA, Cahn P, et al. Antiretroviral treatment of adult HIV infection. JAMA 2010; 304:321.

32. Wolbers M, Bucher H, Furrer H, et al. Delayed diagnosis of HIV infection and late initiation of antiretroviral therapy in the Swiss HIV Cohort Study. HIV Med 2008; 9:397-405.

33. Croxford S, Burns F, Copas A, Pharris A, Rinder Stengaard A, Delpech V. Factors associated with delayed linkage to care following HIV diagnosis in the WHO European Region. HIV Med 2018; 19:40-6.

34. Tavoschi L, Gomes Dias J, Pharris A, et al. New HIV diagnoses among adults aged 50 years or older in 31 European countries, 2004-15: an analysis of surveillance data. Lancet HIV 2017; 4:e514-21.

35. Vo TTN, Ledergerber B, Keiser O, et al. Durability and outcome of initial antiretroviral treatments received during 2000-2005 by patients in the Swiss HIV Cohort Study. J Infect Dis 2008; 197:1685-94.

36. Wagner GJ, Linnemayr S, Ghosh-Dastidar B, Currier JS, Hoffman R, Schneider S Supporting Treatment Adherence Readiness through Training (START) for patients with HIV on antiretroviral therapy: study protocol for a randomized controlled trial. Trials 2016; 17:162.

37. McNabb JJ, Nicolau DP, Stoner JA, Ross J. Patterns of adherence to antiretroviral medications: the value of electronic monitoring. AIDS 2003; 17:1763-7.

38. Glass TR, Cavassini M. Asking about adherence-from flipping the coin to strong evidence. Swiss Med Wkly 2014; 144:1-7.

39. von Wyl V, Klimkait T, Yerly S, et al. Adherence as a predictor of the development of class-specific resistance mutations: the Swiss HIV Cohort Study. PLoS One 2013; $8: 1-11$ 